\title{
Associated Factors in Sarcopenia Secondary to Non-Terminal Chronic Kidney Disease in Older Adults
}

\author{
Carlos Quiñónez-Olivas ${ }^{1 *}$, Ricardo Salinas-Martínez¹, Daniel Gámez-Treviño ${ }^{1}$, Carlos Beltrán-García1, \\ Lorena M. Leyva-Gonzalez ${ }^{1}$ and Omar D. Borjas-Almaguer ${ }^{2}$ \\ ${ }^{1}$ Geriatrics Services; ${ }^{2}$ Department of Internal Medicine. "Dr. José Eleuterio González" University Hospital, Autonomous University of Nuevo León, \\ Nuevo León, Mexico
}

\begin{abstract}
Background: The progressive deterioration of muscle mass (MM), strength, and poor physical performance observed in elderly adults is known as primary sarcopenia. One of the secondary causes is a result of renal disease in the elderly in Mexico. Objective: The primary objective of this study was to determine the factors associated with the secondary sarcopenia to chronic renal disease (CRD) in older adults without dialysis in northeastern Mexico. Methods: Elderly adults over 60 years old, independent in their basic everyday activities, and a filtration rate of $<60 \mathrm{ml} / \mathrm{min} / 1.73 \mathrm{~m}^{2}$ and/or proteinuria $\geq 30 \mathrm{mg}$ were selected randomly from the geriatric outpatient clinic at a university hospital in Mexico. The patients underwent an integral geriatric assessment using different scales, i.e. grip strength, anthropometric, and physical performance. MM was obtained through electric bioimpedance analysis, and a sarcopenia diagnosis was conducted based on the European Working Group on Sarcopenia in Older People algorithm. Results: A total of 84 patients with a mean age of 76 years $( \pm 7.5)$. The prevalence of sarcopenia was $51 \%$. Adjusted logistic regression was the Lawton-Brody index (LBI) odds ratio (OR) 0.574 (Confidence interval $(C I) 95 \%$ 0.364-0.905, $p=0.017$ ). Body mass index (BMI) OR 0.463 (CI 95\% $0.231-0.931, p=0.031$ ). Fat mass (FM) OR 1.41 (Cl 95\% 1.019-1.949, $p=0.038$ ). Age OR 1.16 (Cl 95\% 1.026-1.315, $p=0.018$ ). Conclusion: Independent factors linked to sarcopenia secondary to non-terminal CRD are age, FM, LBI, and BMI in older Mexican adults in northeastern Mexico.
\end{abstract}

Key words: Chronic kidney disease. Sarcopenia. Older adults.

\section{Introduction}

In the United States, estimations suggest that by 2050 , there will be an increase of $20 \%$ (70 million) in individuals over 65 years of age. One in every five adults will be over 65 years of age. Furthermore, there will be an increase in risk factors such as hypertension and diabetes, which increase the prevalence of chronic renal disease (CRD) in older adults ${ }^{1}$.

According to the United States Renal Data System, adults older than 65 years cover the population with the fastest growth of patients with terminal-CRD (t-CRD) and non-t-CRD (NTCRD) CRD2.

The National Health and Nutrition Examination Survey compared CRD prevalence from 1988 to 1994, and there was a significant increase in the prevalence of CRD in adults over 60 years of age, going from a prevalence of $37 \%-47 \%$ in the past 6 years ${ }^{3,4}$.

Since 2013, Kidney Disease: Improving Global Outcomes (KDIGO) has standardized the definitions for CRD; however, definitions do not differentiate CRD from normal renal senescence properly. Hence, there

\section{Correspondence:}

Carlos Quiñónez-Olivas

E-mail: carlos_quinonez@ hotmail.com
Available online: 01-10-2018

Date of reception: 22-10-2017

Date of acceptance: 09-05-2018 DOI: 10.24875/RMU.M18000018
Medicina Universitaria. 2018;20(2):68-77 www.medicinauniversitaria.org

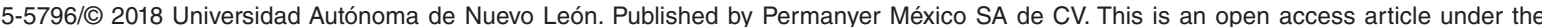
CC BY-NC-ND license (http://creativecommons.org/licenses/by-nc-nd/4.0/). 


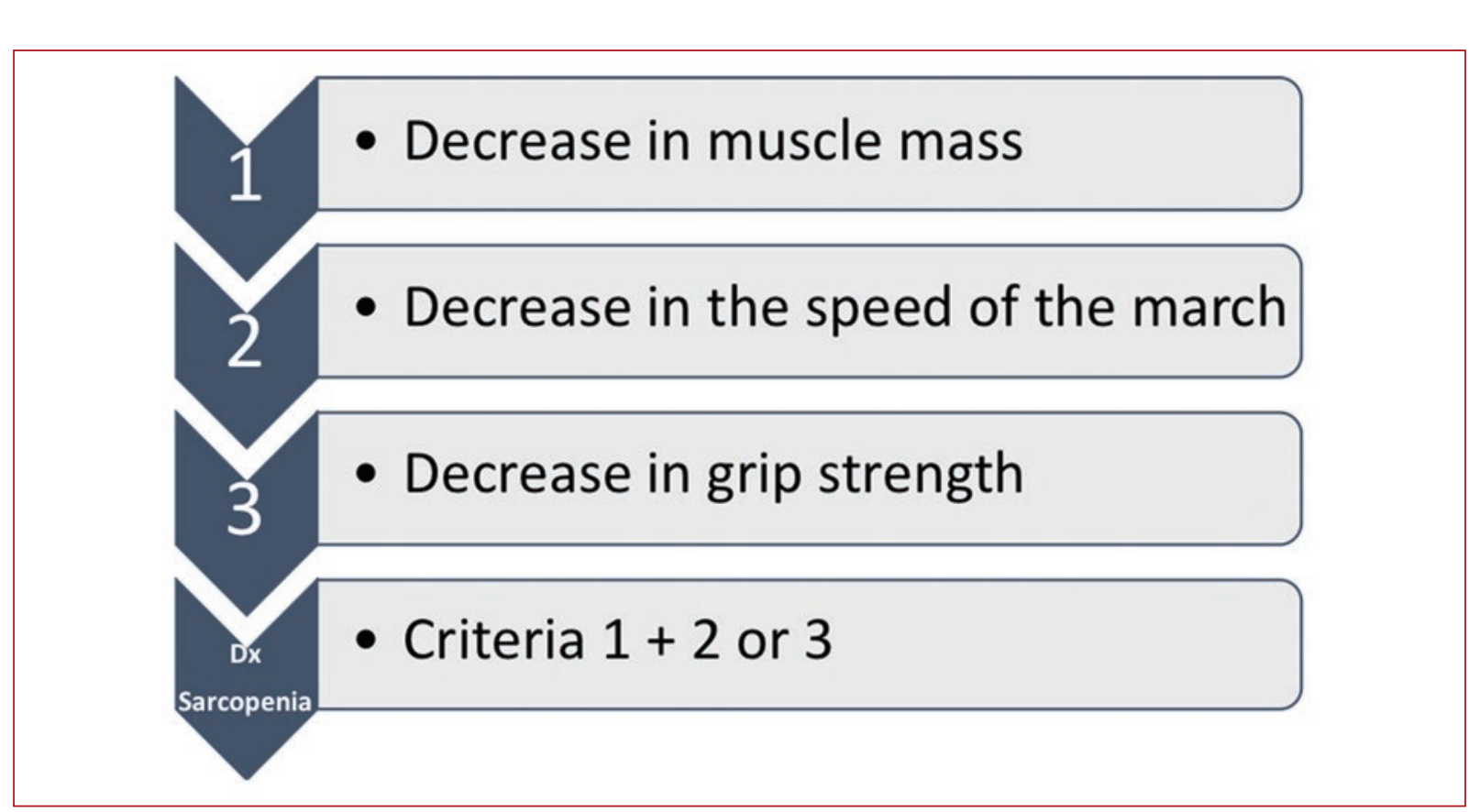

Figure 1. Modified algorithm for the diagnosis of sarcopenia according to the European Working Group on Sarcopenia in Older People ${ }^{10}$.

is a concern of CRD overdiagnosis in older adults. Furthermore, it was acknowledged that Stage 3 CRD is widespread, and many of the complications only occur in those with an estimated glomerular filtering rate (eGFR) of $<45 \mathrm{ml} / \mathrm{min} / 1.73 \mathrm{~m}^{2}$. 5,6

The KDIGO guide did not explicitly recommend a specific formula to calculate eGFR. Nevertheless, today, the only formula validated in older adults is the Berlin initiative study (BIS). Recently, developed equations from BIS may be more precise and accurate tools to estimate eGFR in older adults ${ }^{7,8}$.

The current definition of sarcopenia states that it is a geriatric syndrome characterized by the progressive and generalized loss of skeletal muscle mass (MM) and strength with a risk of adverse results, i.e., physical disability, bad quality of life, and an increase in mortality ${ }^{9}$. The European Working Group on Sarcopenia in Older People (EWGSOP) (Fig. 1) recommends the use of the following criteria - (1) low MM and (2) low muscular function strength measured with the grip or physical performance measured by gait speed for the diagnosis of sarcopenia ${ }^{10}$.

Aging is linked to sarcopenia and a greater CRD prevalence. It is important to highlight the fact that uremic sarcopenia secondary to CRD seems to be the most appropriate term to describe the process of progressive and accumulative loss of MM produced in CRD. Thus, it becomes a primary therapeutic target in the prevention and treatment of MM loss among these patients ${ }^{11,12}$.

Sarcopenia secondary to CRD has several responsible mechanisms such as hormonal imbalance, malnourishment, glycogen exhaustion, improper transportation of oxygen as a result of anemia, metabolic acidosis, and electrolyte disorder ${ }^{13,14}$. This is quite common, with a general prevalence above $50 \%$ in patients undergoing dialysis ${ }^{15,16}$. The objective of this study is to determine factors linked to sarcopenia secondary to CRD in older adults without renal replacement therapy.

\section{Methods}

\section{Design}

We performed a transversal study. For this study, we invited all subjects who attended a consultation at the Geriatrics Department of the "Dr. Jose Eleuterio González" University Hospital, in Monterrey, Nuevo Leon, Mexico, between March and September 2015 to participate. Our study was approved by the Bioethics Committee.

Inclusion criteria were 60 -year-old adults or older, with a filtration rate of $<60 \mathrm{ml} / \mathrm{min} / 1.73 \mathrm{~m}^{2}$ and/or proteinuria $\geq 30 \mathrm{mg} / \mathrm{dl}$, independent and able to perform their basic everyday activities (Katz index [KI] A or $>5$ ) (Annex 1). 
Exclusion criteria were patients with an articular prosthesis, with pacemakers, undergoing androgen replacement therapy, using growth hormones, or steroids in the present year. Furthermore, patients who were hospitalized in the past 3 months, patients with t-CRD (eGFR $<15 \mathrm{ml} / \mathrm{min} / 1.73 \mathrm{~m}^{2}$ ), chronic heart failure NYHA III, chronic obstructive pulmonary disease ORO C or D, CHILD B or C chronic hepatic, moderate cognitive deterioration (mini-mental state examination [MMSE] <15) (Annex 2), use of diuretics, and active use of nonsteroidal anti-inflammatory drugs for more than 2 weeks or with an active urinary tract infection.

For this study, a non-probabilistic sample was determined, including all patients who attended the hospital within the covered period and complied with the inclusion and exclusion criteria.

\section{Intervention and instruments}

After signing an informed consent form, the participants underwent an integral geriatric assessment using the following validated scales (See annexes): KI $(\text { Annex } 1)^{15}$ and Lawton-Brody index (LBI) (Annex 3) ${ }^{16}$, MMSE (Annex 2) ${ }^{17}$, Yesavage's Geriatric Depression Scale (Annex 4) ${ }^{18}$, and the short form Mini Nutritional Assessment (MNAsf) (Annex 5) ${ }^{19}$. Performance was measured by the Short Physical Performance Battery (Annex 6$)^{20}$ and grip strength (GSt) with a dynamometer and gait speed (GSp) in m/s (a 4-m walk). Anthropometric measurements of height, weight, waist/hip index, and calf circumference were taken.

\section{MM and anthropometric measurements}

The measurement of the MM of the whole body was taken with a QUANTUM III Bioelectrical Impedance Analyzer, with the subjects in a supine position and the electrodes placed on the right side of the body in the wrist and ankle (bioimpedance analysis [BIA] Quantum IV, RJL systems). The BIA estimates fat mass (FM) and resistance $(R)$ in Ohms, to later calculate MM with the following formula: ${ }^{21}$

Muscular mass $(\mathrm{kg})=\left[\left(\right.\right.$ height $\left.2 / \mathrm{R}^{*} 0.401\right)+\left(3,825^{*}\right.$ gender $)$ $+($ age $-0.071 \mathrm{X})]+5.102$ Height $=$ centimeters, $\mathrm{R}=$ resistance in Ohms (by BIA), constants: male: 0, female: 1.

Body weight $(\mathrm{kg})$ and height $(\mathrm{cm})$ were measured using the scale meter, and the calf circumference was measured using a Rosscraft metal band. The body mass index $(\mathrm{BMI})=$ Weight $(\mathrm{Kg}) /$ height squared $\left(\mathrm{m}^{2}\right)$, and the skeletal MM index $=$ muscle mass $(\mathrm{kg}) /$ height squared $\left(\mathrm{m}^{2}\right)$.

\section{Grip strength}

This measurement was taken with a digital dynamometer using the dominant hand and by calculating the average of three different occasions, with intervals of $1 \mathrm{~min}$ between each.

\section{The diagnosis of sarcopenia}

This was done based on the EWGSOP algorithm, the cutoff points selected for sarcopenia diagnosis were FP $30 \mathrm{~kg}$ and $<18 \mathrm{~kg}, \mathrm{VM}<0.8$ and $<0.6 \mathrm{~m} / \mathrm{s}$ (Data taken from ENSANUT 2012 and ENASEM) ${ }^{22}$, and IMME $\leq 8.87$ and $\leq 6.42 \mathrm{~kg} / \mathrm{m}^{2}$, in men and women, respectively ${ }^{9}$ (Fig. 2).

\section{Diagnosis of chronic kidney disease (CKD)}

Serum creatinine (SCr) measured in the same year of the study was used with the enzymatic method, and then, the eGFR was calculated using the formula BIS1:3736*creatinine- $0.87 \times$ age $-0.95 \quad(\times 0.82$ if female).

\section{Statistical analysis}

The variables contained in the descriptive statistics were expressed as mean and standard deviation (SD) or median and the interquartile range. The normality of the variables was analyzed by the Kolmogorov-Smirnov test. A univariate analysis was carried out to determine the statistical significance of the comparison variables between the groups with sarcopenia and without sarcopenia (the Student's parametric $t$-test or the non-parametric Mann-Whitney $U$ test were used for the quantitative variables. Along with the $\chi^{2}$ test for the categorical variables and an adjusted logistic regression analysis was carried out for the variables that had a statistical significance in the multivariate analysis. $p=0.05$ or less was considered statistically significant. The SPSS for Windows program (SPSS Inc., Chicago, IL, version 20.0) was used to carry out all statistical tests.

\section{Results}

The total sample was 84 subjects with a mean age of 76 years $( \pm 7.5), 62 \%$ women and $38 \%$ men. The prevalence of sarcopenia was $51 \%$ (43 subjects), $60 \%$ women and $40 \%$ men. Moreover, $49 \%$ (41 subjects) did 


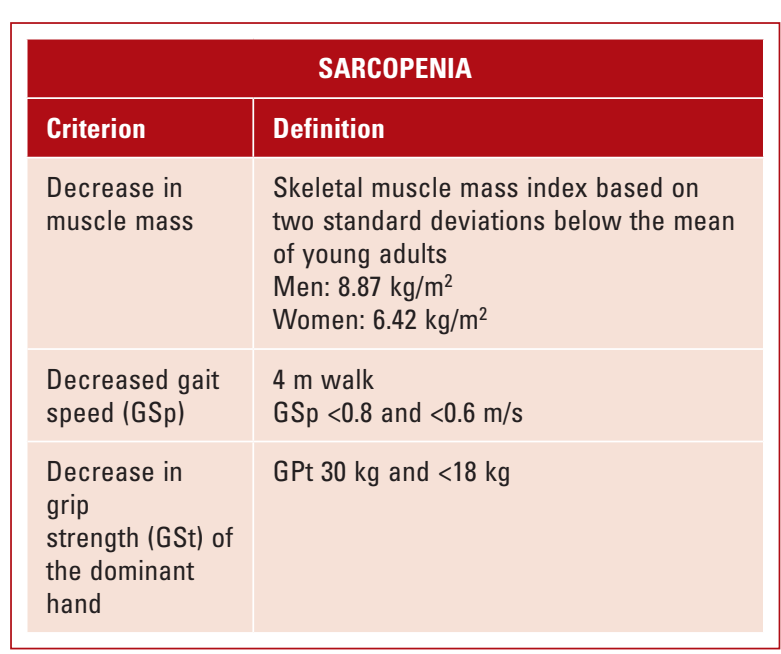

Figure 2. Values of the criteria for the diagnosis of sarcopenia in Mexico. Data obtained from the National Health and Nutrition Survey (ENSANUT 2012) and the National Health and Aging Study in Mexico (ENASEM).

not have sarcopenia, $63 \%$ women and $37 \%$ men. The results of the comparison between the groups with sarcopenia and without sarcopenia for the continuous variables are expressed as mean \pm SD or median interquartile range (Table 1).

Statistically significant differences were found in age $p \leq 0.001$ (95\% confidence interval [Cl] 3.5, -9.4), ILB $p \leq 0.001(95 \% \mathrm{Cl}-2.0,-6.4)$, BMI $p \leq 0.001(95 \% \mathrm{Cl}$ $-7.1,-3.3), \mathrm{CP} p \leq 0.001$ (95\% Cl $-7.4,-2.9)$, MG $p=0.024(95 \% \mathrm{Cl}-7.3,-0.53)$, and $\mathrm{CrS} p=0.046 \mathrm{IC}$ $(95 \% \mathrm{Cl}-0.7094,0.000)$, and there was no statistical significance in the MMSE, MNAsf, or Charlson comorbidity index. A higher percentage of patients with diabetes and hypertension was found in non-sarcopenic patients but without being statistically significant. Subjects with sarcopenia have a significant loss in the ability to perform daily activities (LBI), and they also have a lower BMI compared to subjects without sarcopenia. The anthropometric markers of body composition such as calf circumference and those measured by bioimpedance as FM also had statistically significant differences, which was to be expected in patients with sarcopenia (Table 2). Finally, when performing the multivariate analysis by means of logistic regression, the independent factors associated with sarcopenia secondary to CKD were significantly LBI (odds ratio [OR] $0.574,95 \% \mathrm{Cl}$ $0.364,0.905, p=0.017$ ), BMI (OR 0.463; 95\% Cl 0.231, 0.931, $p=0.031$ ), FM (OR $1.4195 \% \mathrm{Cl} 1.019,1949$, $p=0.038)$, and age (OR $1.1695 \% \mathrm{Cl} 1.026,1.315$, $p=0.018$ ) (Table 3). These findings demonstrate that
Table 1. Anthropometric measures, physical performance, and body composition of the patients included in the study.

\begin{tabular}{|l|c|c|}
\hline $\boldsymbol{n}=\mathbf{8 4}$ & Average & \pm SD (IOR) \\
\hline Height $(\mathrm{cm})$ & 155.4 & \pm 9.8 \\
\hline Weight $(\mathrm{Kg})$ & 67.3 & \pm 15.7 \\
\hline BMI $\left(\mathrm{kg} / \mathrm{m}^{2}\right)$ & 27.7 & \pm 5.1 \\
\hline Calf circumference $(\mathrm{cm})$ & 33.5 & $(31$ a 36$)$ \\
\hline Gait speed $(\mathrm{m} / \mathrm{s})$ & 0.70 & \pm 0.26 \\
\hline Grip strength $(\mathrm{Kg})$ & 20.4 & \pm 8.3 \\
\hline SPPB & 7.1 & \pm 2.8 \\
\hline Muscle mass by BIA $(\mathrm{Kg})$ & 17.3 & \pm 5.9 \\
\hline Fat mass $(\mathrm{kg})$ & 21.3 & \pm 8.0 \\
\hline Muscle mass index $\left(\mathrm{Kg} / \mathrm{m}^{2}\right)$ & 6.99 & \pm 1.7 \\
\hline
\end{tabular}

*SD: standard deviation, IQR: interquartile range, SPPB: short physical performance battery. BMI: body mass index BIA: bioimpedance analysis

the $\mathrm{LBI}$ and $\mathrm{BMI}$ were associated with an increased likelihood of sarcopenia ( $43 \%$ and $54 \%$, respectively), but FM and age were associated with a lower likelihood of sarcopenia ( $16 \%$ and $41 \%$, respectively).

\section{Discussion and Conclusion}

Our results suggest that the factors linked to sarcopenia secondary to $\mathrm{CRD}$ are different from the glomerular filtrated rate calculated by $\mathrm{SCr}$, which has only been described in a Korean population, without showing any statistical significance ${ }^{23}$. The latter can be explained because the creatinine serum was significantly lower in sarcopenic patients, which means that, in this study, it acted as a subrogated marker of MM. The KDIGO guides of 2013 suggest that in older adults with CRD calculated by SCr, cystatin C ought to be used as well to verify renal function, which we were unable to verify in our study due to a lack of economic resource and the unavailability of this reactive in Mexico ${ }^{5,24}$.

Malnutrition measured by the MNAsf scale among patients with and without sarcopenia did not show a statistical significance, yet both groups had a mean value under 11 points, which puts them at high risk and high probability of developing malnutrition. In the council of the International Society of Renal Nutrition and Metabolism, the authors observed that malnutrition linked to CRD is caused by lack of appetite. Nevertheless, 
Table 2. Comparison of groups with and without sarcopenia.

\begin{tabular}{|c|c|c|c|c|}
\hline Variables & $\begin{array}{c}\text { Sarcopenia } \\
\qquad n=43\end{array}$ & $\begin{array}{c}\text { No sarcopenia } \\
\qquad n=41\end{array}$ & \multicolumn{2}{|l|}{$p$ value } \\
\hline Age (years) & $79.4 \pm 7.1$ & $72.9 \pm 6.6$ & $<0.001$ & $\stackrel{\odot}{\sim}$ \\
\hline Height (cm) & $154.3 \pm 9.2$ & $156.6 \pm 10.5$ & 0.292 & $\stackrel{1}{2}$ \\
\hline Weight (Kg) & $60.3 \pm 11.8$ & $74.6 \pm 16.1$ & $<0.001$ & हृ \\
\hline $\mathrm{BMI}\left(\mathrm{kg} / \mathrm{m}^{2}\right)$ & $25.2 \pm 3.6$ & $30.4 \pm 5.1$ & $<0.001$ & $\frac{1}{2}$ \\
\hline Calf circumference $(\mathrm{cm})$ & $32(30$ a 34$)$ & $35(33$ a 38$)$ & $<0.001$ & . \\
\hline SPPB & $6.7 \pm 2.7$ & $7.6 \pm 2.8$ & 0.107 & $\frac{\check{1}}{(1}$ \\
\hline Fat mass $(\mathrm{kg})$ & $19.4 \pm 6.2$ & $23.4 \pm 9.2$ & 0.024 & $\frac{\curvearrowleft}{2}$ \\
\hline Muscular mass index $\left(\mathrm{kg} / \mathrm{m}^{2}\right)$ & $6.1 \pm 1.0$ & $7.9 \pm 1.9$ & $<0.001$ & 을 \\
\hline Serum creatinine (mg/dl) & 1.1 (1 a 1$)$ & $1.2(1$ a 2$)$ & 0.046 & $\frac{c}{4}+$ \\
\hline TFGe by BIS1 (ml/min/1.73m²) & 48 (38 a 57$)$ & 48 (32 a 55$)$ & 0.525 & $\frac{c}{0}$ \\
\hline Abnormal *ILB (\%) & 15 & 13 & *0.954 & $\frac{n}{\varepsilon}$ \\
\hline Abnormal *MMSE (\%) & 14 & 12 & $* 0.887$ & 닐 \\
\hline Abnormal *GDS $(\%)$ & 11 & 10 & *0.946 & $\frac{c}{d}$ \\
\hline Abnormal *MNAsf (\%) & 2 & 1 & ${ }^{*} 0.689$ & $\frac{\Sigma}{3}$ \\
\hline${ }^{*} \mathrm{DM} / \mathrm{SAH} /$ Charlson index $>3(\%)$ & $(44 / 65 / 90)$ & $(46 / 70 / 92)$ & $* 1.000$ & 은 \\
\hline $\begin{array}{l}\text { nterpretation outside normal range: LBI: La } \\
\text { DSS: geriatric depression scale (abnormal > } \\
\text { AH: systemic arterial hypertension; }{ }^{*} \chi^{2} \text { witl }\end{array}$ & $\begin{array}{l}5 \text { points); } M \\
\text { nal assessm }\end{array}$ & $\begin{array}{l}\text { examination (abn } \\
\text { al }<8 \text { points); DM }\end{array}$ & & 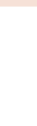 \\
\hline
\end{tabular}

Table 3. Independent factors associated with sarcopenia secondary to chronic kidney disease* ${ }^{*}$.

\begin{tabular}{|l|l|l|l|l|}
\hline $\begin{array}{l}\text { Variables with } \\
\text { statistical } \\
\text { significance }\end{array}$ & $p$-value & OR & \multicolumn{2}{|c|}{$95 \%$ CI } \\
\hline LBI & 0.017 & 0.574 & 0.364 & 0.905 \\
\hline Age (years) & 0.018 & 1.161 & 1.026 & 1.315 \\
\hline BMI (Kg/m $\left.{ }^{2}\right)$ & 0.031 & 0.463 & 0.231 & 0.931 \\
\hline CC (cm) & 0.126 & 0.789 & 0.582 & 1.069 \\
\hline FM (Kg) & 0.038 & 1.409 & 1.019 & 1.949 \\
\hline SCr (mg/dl) & 0.419 & 0.602 & 0.176 & 2.059 \\
\hline
\end{tabular}

*Logistic regression adjusted with the variables that had statistical significance: Lawton-Brody index (LBI), age, body mass index (BMI), calf circumference (Cp), fat mass (MG), serum creatinine ( $\mathrm{SCr}$ ). OR: odds ratio, $\mathrm{Cl}$ : confidence interval

other factors are required to complete the diagnosis of sarcopenia syndrome, among these being: acidemia, resistance to insulin, lowering of the growth hormone and growth factor similar to insulin, hyperglucagonemia, and hyperparathyroidism. Yet these alterations are more prevalent in terminal stages of the disease ${ }^{25}$. Our study excluded patients in terminal stage CRD, which could explain the leading cause for the lack of statistical difference among the groups with and without sarcopenia in the MNAsf scale.

MMSE did not show a statistical significance between the compared groups, yet the mean MMSE values corresponded to a mild cognitive deterioration. CRD has been described to have a linear relationship with vascular cognitive deterioration, even adjusted by multiple risk factors. This low average in the MMSE in our study may very well be explained by the presence of vascular cognitive deterioration in patients ${ }^{26}$.

The main limitation was due to the fact that the renal function calculation was performed through SCr. Ideally, we should have complemented it by performing eGFR with cystatin $C$ to minimize classification errors between renal senescence and CRD. The lack of availability of cystatin $C$ in Mexico made it impossible for us to include it in this study. Another relevant limitation was that the vascular etiology of the cognitive deterioration was not verified due to a lack of cerebral imaging studies, a result of the scarce economic resources. 
In conclusion, in the present study, we were able to demonstrate a high prevalence of $51 \%$ of sarcopenia secondary to CRD in older adults in northeast Mexico. The results of the factors linked to sarcopenia secondary to NTCRD in older patients are age, BMI, adiposity measured by FM, and functionality measured by everyday activities with the LBI. These associations are relevant since they tell us that older adults with NTCRD of lesser age and with lower adiposity have a lower probability of suffering from sarcopenia. On the other hand, those with less functionality in instrumented activities in the LBI and a low BMI will have a greater probability of sarcopenia.

\section{Ethical disclosures}

Protection of human and animal subjects. The authors declare that no experiments were performed on humans or animals for this study.

Confidentiality of data. The authors declare that they have followed the protocols of their work center on the publication of patient data.

Right to privacy and informed consent. The authors have obtained the written informed consent of the patients or subjects mentioned in the article. The corresponding author is in possession of this document.

\section{References}

1. From the Centers for Disease Control and Prevention. Public health and aging: trends in aging-united states and worldwide. JAMA. 2003;289: 1371-3.

2. Paniagua R, Ramos A, Fabian R, Lagunas J, Amato D. Chronic kidney disease and dialysis in Mexico. Perit Dial Int. 2007;27:405-9.

3. Antonio MD, Francisco MB, Teresa TY, Angélica MM, Leticia AS. Epidemiología de la insuficiencia renal crónica en México. Dial Traspl. 2010;31:7-11.

4. Centers for Disease Control and Prevention (CDC). National Center for Health Statistics (NCHS). National Health and Nutrition Examination Survey Data. Hyattsville, MD: U.S. Department of Health and Human Services, Centers for Disease Control and Prevention; 1988-1994.
5. Eknoyan G, Lameire N, Eckardt KU, et al. KDIGO 2013 board members: clinical practice guideline for the evaluation and management of chronic kidney disease. Kidney 冈nt Org. 2013;3:136-50.

6. Maw TT, Fried L. Chronic kidney disease in the elderly. Clin Geriatr Med. 2013;29:611-24

7. Levey AS, de Jong PE, Coresh J, et al. The definition, classification, and prognosis of chronic kidney disease: a KDIGO controversies conference report. Kidney Int. 2011;80:17-28.

8. Schaeffner ES, Ebert N, Delanaye P, et al. Two novel equations to estimate kidney function in persons aged 70 years or older. Ann Intern Med. 2012;157:471-81.

9. Baumgartner RN, Koehler KM, Gallagher D, et al. Epidemiology of sarcopenia among the elderly in new Mexico. Am J Epidemiol. 1998;147:755-63.

10. Cruz-Jentoft AJ, Baeyens JP, Bauer JM, et al. Sarcopenia: European consensus on definition and diagnosis: report of the european working group on sarcopenia in older people. Age Ageing. 2010;39:412-23.

11. Workeneh BT, Mitch WE. Review of muscle wasting associated with chronic kidney disease. Am J Clin Nutr. 2010:91:1128S-32S.

12. Stenvinkel $P$, Alvestrand $A$. Inflammation in end-stage renal disease: sources, consequences, and therapy. Semin Dial. 2002;15:329-37.

13. Leavey SF, Weitzel WF. Endocrine abnormalities in chronic renal failure. Endocrinol Metab Clin North Am. 2002;31:107-19.

14. Diesel W, Emms M, Knight BK, et al. Morphologic features of the myopathy associated with chronic renal failure. Am J Kidney Dis. 1993;22:677-84.

15. Katz S, Ford AB, Moskowitz RW, Jackson BA, Jaffe MW. Studies of illness in the aged: the index of ADL: a standardized measure of biological and psychosocial function. JAMA. 1963;185:914-19.

16. Lawton MP, Brody EM. Assessment of older people: self-maintaining and instrumental activities of daily living. Gerontologist. 1969;9:179-86.

17. Folstein M, Folstein SE, McHugh PR, et al. "Mini-mental state" a practical method for grading the cognitive state of patients for the clinician. $J$ Psychiatr Res. 1975;12:189-98.

18. Yesavage JA, BrinK TL, Rose TL, et al. Development and validation of a geriatric depression scale: a preliminary report. J Psychiatr Res. 1983:17:37-49.

19. Kaiser MJ, Bauer JM, Ramsch C, et al. Validation of the mini nutritional assessment short-form (MNAA (R)-SF): a practical tool for identification of nutritional status. J Nutr Health Aging. 2009;13:782-8.

20. Guralnik JM, Simonsick EM, Ferrucci L, et al. A short physical performance battery assessing lower extremity function: association with self-reported disability and prediction of mortality and nursing home admission. J Gerontol Med Sci. 1994;49:M85-94.

21. Janssen I, Heymsfield SB, Baumgartner RN, Ross R. Estimation of skeletal muscle mass by bioelectrical impedance analysis. J Appl Physiol. 2000;89:465-71.

22. Gutiérrez-Robledo LM, López-Teros MT, Cruz-Arenas E, Robles-Jiménez L. P6-cut-off values for gait speed and handgrip strength in Mexican older adults. J Frailty Aging. 2015;1:47.

23. Moon SJ, Kim TH, Yoon SY, Chung JH, Hwang HJ. Relationship between stage of chronic kidney disease and sarcopenia in Korean aged 40 years and older using the Korea national health and nutrition examination surveys (KNHANES IV-2, 3, and V-1, 2), 2008-2011. PLoS One. 2015;10:e0130740.

24. Inker $\mathrm{LA}$, Schmid $\mathrm{CH}$, Tighiouart $\mathrm{H}$, et al. Estimating glomerular filtration rate from serum creatinine and cystatin C. N Engl J Med. 2012;367:20-9.

25. Fouque D, Kalantar-Zadeh K, Kopple J, et al. A proposed nomenclature and diagnostic criteria for protein-energy wasting in acute and chronic kidney disease. Kidney Int. 2008;73:391-8.

26. Khatri M, Nickolas T, Moon YP, et al. CKD associates with cognitive decline. J Am Soc Nephrol. 2009;20:2427-32. 


\section{Annexes}

Annex 1. Clinimetry: Katz index of basic activities of daily life Katz index.

\section{Bathroom}

Independent: Needs help to wash a single part (such as your back or an incapacitated limb) or can bathe completely without help. Dependent: Needs help to wash more than one part of the body, to get out or enter the bathtub, or does not wash alone.

\section{Dress}

Independent: Takes clothes alone and puts them on, puts on ornaments and coats and can use zippers (shoes are not tied). Dependent: Does not dress alone or remains partially dressed.

\section{Use of the toilet}

Independent: Access to the toilet, in and out of it, the excretory organs are cleaned and the clothes are fixed (can use mechanical supports or not).

Dependent: Uses a potty or wedge or precise help to access the toilet and use it.

\section{Mobility}

Independent: Can enter and leave the bed and sit and stand up from a chair alone (with mechanical supports or not). Dependent: Needs help to use the bed and/or chair; does not make one or more trips.

\section{Continence}

Independent: Complete control of urination and defecation.

Dependent: Partial or total urinary or fecal incontinence.

\section{Feeding}

Independent: Take the food from the plate or its equivalent to the mouth (excluding cutting meat and butter or other foods). Dependent: Needs help for the action of feeding or needs enteral or parenteral feeding.

Normal interpretation $>5$

Annex 2. Mini-mental state examination.

\section{Mini*mental (Folstein)}

Which year is it?

Which season of the year is it?

What is the date today?

Which month?

What is the day of the week?

Which hospital (place) are we in?

Which floor (area, service)?

Which town (city)?

Which province?

Which country?

Name three words:

(1). Cent (2). Horse (3). Apple, then the patient is asked to repeat them.

This first repetition grants scoring. Grant 1 point per each correct word.

\section{Score}

\section{Domains}

Temporal orientation (5)

Spatial orientation (5)

Fixation (3)

Subtractions sequences 100-7: 93, 86,79,72,65

Or spell world backward

d__ I____ o__ w

Remember these three words. 1 point per each word. ip. 
Annex 2. Mini-mental state examination. (Continued)

\section{Mini*mental (Folstein)}

Denomination - show a watch and a pen and ask: what is this?

1 point $=$ watch 1 point $=$ pen

Repetition - ask the patient to repeat "nor yes, nor "no," nor "but"

Order: Now ask the patient to perform the following: take this piece of paper with your right hand, fold it in half, and put it on the desk. 1 point $=$ grab it with the right hand, 1 point $=$ fold it in half, and 1 point $=$ place it on the desk.

Writing: Have the patient write a phrase (with subject and predicate) $=1$ point Copy: Draw the following pentagons.

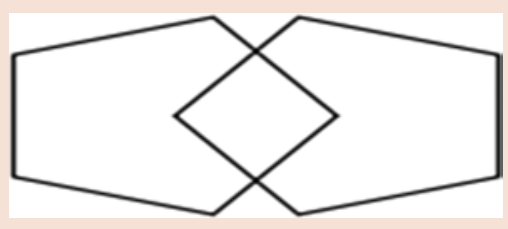

Interpretation normal $>23$

\section{Score} Domains

Language (9)

Annex 3. Lawton and Brody instrumental activities of daily living scale.

Lawton-Brody

Ability to use the telephone

Use the telephone on their own initiative

Is able to dial some familiar numbers well

Is able to answer the phone but not dial

Cannot use the phone

Shopping

Can make all necessary purchases independently

Can do small things independently

Needs to be accompanied to make any purchase

Totally incapable of buying anything

Food preparation

Organizes, prepares, and serves meals by themselves properly

Can prepare meals properly if ingredients are provided

Prepares, warms, and serves food but does not follow a proper diet

Needs food served and prepared

House care

Maintains the house alone with occasional help

Does light tasks such as washing dishes or making beds

Performs light tasks but cannot maintain an adequate level of cleanliness

Needs help in all housework

Does not participate in any housework

Washing clothes

Washes all their clothes by themselves

Washes their underclothes by themselves

All laundry must be done by another

Use of means of transportation

Travels alone on public transport or drives their own car

Is able to take a taxi but does not use any other means of transportation

Can travel by public transport when accompanied by another person

Can use the taxi or the car alone with the help of others

Does not travel at all

Responsibility regarding their medication

Is able to take their medication at the correct time and dose

Takes their medication if the dose was previously prepared

Is not able to take their medication

Managing their economic affairs

Takes care of their economic affairs on their own

Can make everyday purchases, but needs help with large purchases and banks

Unable to handle money

Normal interpretation $>5$ 
Medicina Universitaria. 2018;20

Annex 4. Yasavage GDS.

GDS

Are you satisfied with your life?

Have you given up many activities?

Do you feel that your life is empty?

Are you often bored?

Are you often in good spirits?

Are you afraid something bad will happen to you?

Do you feel happy most of the time?

Do you often feel abandoned?

Do you prefer to stay at home to go out?

Do you think you have more memory problems than most people?

Do you think it is wonderful to live?

Do you have trouble starting new projects?

Do you feel full of energy?

Do you feel that your situation is desperate?

Do you think that many people are better than you?

GDS: geriatrics depression scale. Norma interpretation $<6$

Annex 5. MNA sf.

MNA short form

A. Has eaten less due to lack of appetite, digestive problems, chewing, or swallowing difficulties in the past 3 months

Eaten a lot less

Eaten less

Eaten the same

B. Recent weight loss $(<3$ months) Weight loss $>3 \mathrm{~kg}$

Unknown

Weight loss between 1 and $3 \mathrm{~kg}$

There has been no weight loss

C. Mobility

From the bed to the couch

Autonomous indoors

Goes outside

D. Have you had an acute illness or psychologically stressful situation in the past 3 months?

Yes

No

0

2

E. Neuropsychological problems Dementia or severe depression

Moderate dementia

Without psychological problems

\begin{tabular}{|c|c|}
\hline 0 & \\
\hline 1 & 잉 \\
\hline 2 & 1 \\
\hline 3 & 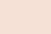 \\
\hline & है \\
\hline 0 & 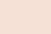 \\
\hline 1 & 0 \\
\hline 2 & (]) \\
\hline & $\frac{\overline{0}}{3}$ \\
\hline 0 & $\frac{\varkappa}{c}$ \\
\hline 2 & $\Phi$ \\
\hline & 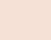 \\
\hline 0 & 잉 \\
\hline 1 & 0 \\
\hline 2 & \\
\hline
\end{tabular}

(Continued) 
Annex 5. MNA sf. (Continued)

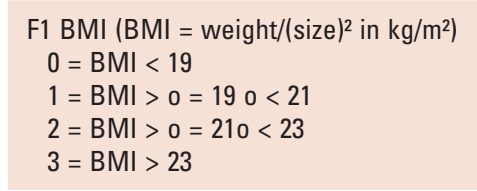

In case of not having BMI to answer F2/do not answer F2 if you have contemplated F1 F2 calf circumference (in $\mathrm{cm}$ )

$$
\begin{aligned}
& 0 \leq 31 \\
& 3=>0=31
\end{aligned}
$$

Total

Interpretation normal >11

8-11: risk of malnutrition

$0-7$ : malnutrition

MNAsf: mini nutritional assessment short form

Annex 6. Short physical performance battery. Interpretation normal $>9$.

\section{Short physical performance battery}

1.

Balance Tests

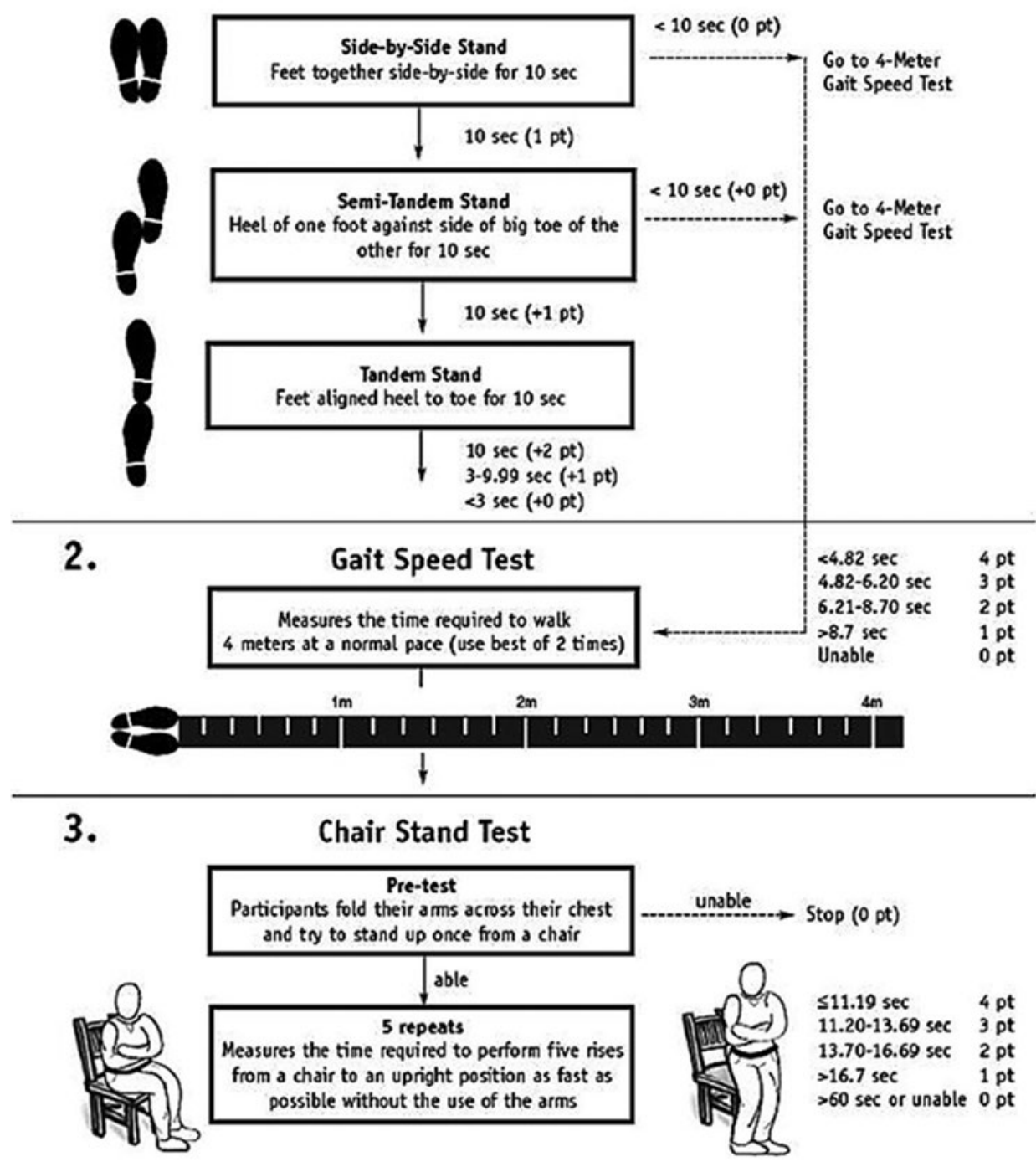

